\title{
Efficacy and safety of intravesical fibrin glue instillation for management of patients with refractory hemorrhagic cystitis: 12-months results. A promising therapy for hemorrhagic cystitis
}

\author{
Alessandra Cassani ${ }^{1}$, Michele Marchioni ${ }^{1}$, Francesco Silletta ${ }^{1}$, Carlo D’Orta ${ }^{1}$, Giulia Primiceri ${ }^{1}$, \\ Ambra Rizzoli ${ }^{1}$, Patrizia Di Gregorio ${ }^{2}$, Sandra Verna ${ }^{2}$, Annalisa Natale ${ }^{3}$, Stella Santarone ${ }^{3}$, \\ Francesco Berardinelli ${ }^{1}$, Luigi Schips ${ }^{1}$ \\ 1 "G. D'Annunzio" University of Chieti, Dept. of Medical, Oral and Biotechnological Sciences, "SS. Annunziata" Hospital, \\ Urology Unit, Chieti, Italy; ASL Abruzzo 2, Department of Urology, Chieti, Italy; \\ 2 "SS. Annunziata" Hospital, Chieti, Italy; ASL Abruzzo 2, Servizio di Medicina Trasfusionale ed Ematologia Aziendale Ospedaliero - \\ Centro Emofilia 52, Chieti, Italy; \\ ${ }^{3}$ Santo Spirito Hospital, Pescara, Department of Hematology, Bone Marrow Transplant Center, Pescara, Italy.
}

\section{Summary Objectives: Fibrin glue (FG) endo-vesical application seems to be a promising therapy} for hemorrhagic cystitis (HC). We aimed to evaluate efficacy and safety of FG instillation in patients with HC.

Methods: Patients with HC not responsive to conventional treatments (bladder irrigation, catheterization, blood transfusions, hyperhydration and endoscopic coagulation) were treated with FG endo-vesical instillation (April 2017- December 2018).

FG was prepared from $120 \mathrm{~mL}$ of patient blood with the Vivostat ${ }^{\circledR}$ system. After standard cystoscopy, bladder was insufflated with carbon dioxide $\left(\mathrm{CO}_{2}\right)$ according to bladder compliance and autologous FG was applied to bladder wall and bleeding sites.

Results: Ten patients included with grade 2 or higher HC secondary to bone marrow graft for hematological diseases (30\%) or to actinic cystitis caused by prostate cancer radiotherapy (RT) (70\%). The median HC onset time after RT was 4.8 (IQR 3.96.3) years and 35 (IQR 27.5-62.5) days after hematopoietic stem cell transplantation (HSCT). Five patients had a complete response after one treatment, three patients had clinical response (grade $<2$ hematuria, amelioration of symptoms), one of them required catheterization and bladder irrigation. One patient required a second instillation of FG achieving a clinical response. No adverse events related to the procedure were recorded, however one patient died for causes not related to the procedure. Median Interstitial Cystitis Symptoms Index was 13.0 (IQR 11.0-15.0) pre-operatively and 4.0 (IQR 2.0-5.0) post-operatively.

Conclusions: Our study showed that, even in hematological patients, autologous FG instillation maybe a safe, repeatable and effective treatment modality in patients with refractory $\mathrm{HC}$.

KEY WORDS: Cystitis; Radiation; Radiotherapy; Hemorrhagic cystitis; Hemorrhagic cystitis therapy; Fibrin glue therapy; Actinic cystitis; HSCT induced cystitis; Hematuria; Allogenic transplant.

Submitted 18 January 2021; Accepted 10 March 2021

\section{INTRODUCTION}

Hemorrhagic cystitis (HC) is a condition defined as the presence of hematuria and lower urinary tract symptoms (LUTS), irritative voiding symptoms, such as urgency, frequency, nocturia and pain or burning with urination (1). Radiotherapy (RT) for pelvic malignancies, including prostate cancer $(2,3)$, could be associated with the development of HC in up to $6.5 \%$ of patients, as result of bladder wall modification and neovascularization (4).

However, HC may be a complication of hematological cancer treatment, too. Medication toxicity and immunemediated hypersensitivity may lead to LUTS and bladder mucosae bleeding in 7 to $68 \%$ of patients treated with cyclophosphamide (5). In literature there are many possible treatments for HC. First line treatments are the most conservative and include hyperhydration, blood transfusion, transurethral three-way catheterization with continuous bladder irrigation, hyaluronic acid instillation and reversal of anticoagulation. Second line treatments also include endo-vesical instillation of several compounds (e.g. aluminum compounds, silver or formalin) as well as transurethral surgery with laser or fulguration. In case of failure, third line options proposed include hyperbaric oxygen (HBO) or arterial embolization. In extreme cases a radical cystectomy with urinary derivation might be indicated (6-10). Despite the variety of treatments available there is not a consensus about the best treatment to use in these cases (11). More recently, FG endo-vesical application seems to be a promising therapy for $\mathrm{HC}(12,13)$. However, literature is limited to only two single center experiences $(12,13)$. Thus, we aim to report results about efficacy and safety of FG therapy in patients with HC.

\section{MATERIALS AND METHODS}

Patients and study population

We reviewed prospectively collected data from our insti- 
tutional dataset about patients with HC treated with FG between April 2017 to December 2018. Our analyses included data about patients' demographic (age, gender), condition that led to the hematuria (RT for prostate cancer, allogenic transplant for Acute lymphoblastic leukemia, Acute myeloid leukemia, Hodgkin's lymphoma), time of hematuria onset (identified as the first episode of hematuria after RT or Hematopoietic stem cell transplantation (HSCT) after systemic chemotherapy treatment with Busulfan $12.8 \mathrm{mg} / \mathrm{kg}$, Thiotepa $10 \mathrm{mg} / \mathrm{kg}$, Fludarabine $150 \mathrm{mg} / \mathrm{m}^{2}$ ), RT type, treatment characteristics (management of the hematuria before the FG instillation), clinical response and toxicity.

Before treatment, all patients had urine culture test, bladder ultrasound, urine analysis, blood test and cystoscopy. All patients had HC grade 2 (8) or more no responsive to conventional therapy, namely catheterization with bladder irrigation, blood transfusions, hyperhydration and endoscopic coagulation. HC was classified in 4 stages. The subgroup of patients who developed HC after radiotherapy was also classified with the European organization for research and treatment of cancer/Radiation therapy oncology Group (RTOG/EORTC) grading of hematuria events due to radiotherapy (14). Main Classification are reported in Table 1.

The bladder capacity was evaluated during pre-operative cystoscopy identifying as maximum capacity the filling volume at which the patient felt pain. All the procedures were performed in operative room under loco-regional anesthesia. Patients with positive urine cytology, bladder neoformation identified by imaging or during cystoscopy, with a suspect upper urinary tract tumor or any suspect of urothelial carcinoma in situ were considered not-eligible for the procedure and were not included in the current study.

\section{Surgical procedure}

We performed a standard cystoscopy with an Iglesias 22 ch cystoscope evaluating the bladder mucosa to rule out eventual bladder tumors and identify mucosal lesions. Emptied bladder is then insufflated with carbon dioxide $\left(\mathrm{CO}_{2}\right)$ up to obtain a good distention of bladder wall, approximately 180-240 cc occur, according to bladder compliance.

No continuous pressure flow was used, and small adjustment were possible thank to the use of a syringe filled with ambient air. Good bladder distention is necessary to allow air spray of the FG, guarantee a good vision and prevent bleeding. Under vision, the FG applicator is introduced through the cystoscope and autologous FG is applied to the bladder wall with specific care of bleeding sites.

The FG instillation procedure usually last approximately 30 minutes. Fibrine Glue (FG) was prepared with the Vivostat $^{\circledR}$ system (Vivolution A/S, Birkerod, Denmark). Vivostat $^{\circledR}$ is automated medical device for production and application of a fibrine sealant, it produces protein components of fibrine gel Sealant from patient' own plasma with no risk of infections related to non-autologous plasma sources.

The Vivostat ${ }^{\circledR}$ system creates from $120 \mathrm{ml}$ of the patient' blood a concentrated fibrine I solution. The solution is then applied with a pH 10 neutralizing solution in a 7:1 ratio to produce fibrine sealant. The process takes 30 minutes to obtain a syringe of fibrin I solution at a PH of 4.4. The Vivostat ${ }^{\circledR}$ application system is composed by an applicator (the mechanical and electronic working part of the system) and a single-use Spraypen ${ }^{\circledR}$. When activated fibrin I and neutralizing solution are delivered in a stream of compressed air forming a fine low-pressure spray of fibrin sealant $(12,13,15)$.

\section{Outcomes measurement}

Patients response was classified as: complete response (no hematuria and no LUTS); clinical response (absence or amelioration of dysuria, urgency, and frequency, no further need of analgesic medication, persistent hematuria grade $<2$ ) and no response (no clinical response, persistent hematuria grade $\geq 2$ ). Full blood count was performed preoperatively and on day 1 to 3. Each patient was investigated with Interstitial Cystitis Symptoms Index (ICSI) (16) pre and postoperative at follow-up. Patients were followed by telephone at 3 and 12 months to assess ICSI and their bladder status.

\section{Statistical analyses}

Descriptive analyses were employed. Qualitative variables were reported as absolute and relative frequencies (\%). Quantitative variables were reported as median and interquartile range (IQR). Wilcoxon test for paired data tested the hypothesis that the median ICSI was higher before the treatment than after.

A level of statistical significance was set at $\mathrm{p}<0.05$. Analyses were performed using the R software environment for statistical computing and graphics (version 3.6.1; http://www.rproject.org/).

\section{Table 1.}

Classification systems.

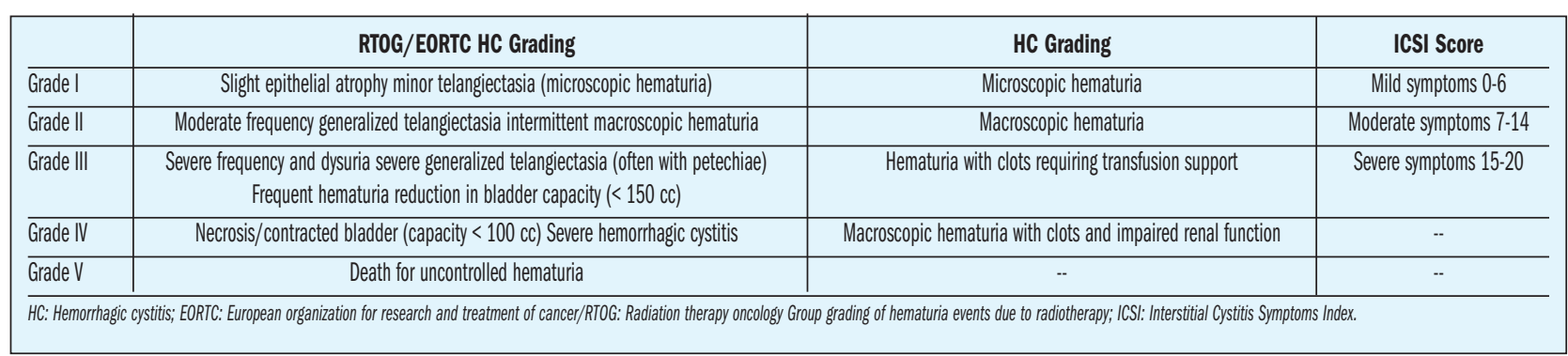


Table 2.

Pre- operative and post- operative (at discharge) patients' blood count.

\begin{tabular}{|c|c|c|c|c|c|c|c|c|}
\hline & \multicolumn{4}{|c|}{ Preoperatively } & \multicolumn{4}{|c|}{ Postoperatively } \\
\hline & $\begin{array}{c}\text { Red blood cells } \\
\text { count }\left({ }^{*} 10^{6} / \mathrm{mm}^{3}\right)\end{array}$ & $\begin{array}{l}\text { Hemoglobin } \\
(\mathrm{g} / \mathrm{dl})\end{array}$ & $\begin{array}{c}\text { Hematocrit } \\
(\%)\end{array}$ & $\begin{array}{c}\text { Platelets count } \\
\left({ }^{*} 10^{3} / \mathrm{mm}^{3}\right)\end{array}$ & $\begin{array}{l}\text { Red blood cells } \\
\text { count }\left({ }^{*} 10^{6} / \mathrm{mm}^{3}\right)\end{array}$ & $\begin{array}{l}\text { Hemoglobin } \\
(\mathrm{g} / \mathrm{dl})\end{array}$ & $\begin{array}{c}\text { Hematocrit } \\
(\%)\end{array}$ & $\begin{array}{l}\text { Platelets count } \\
\left({ }^{*} 10^{3} / \mathrm{mm}^{3}\right)\end{array}$ \\
\hline 1 & 4.74 & 13.8 & 48 & 201 & 4.5 & 13.7 & 43 & 150 \\
\hline 2 & 4.04 & 12.8 & 44 & 220 & 4 & 12.4 & 40 & 200 \\
\hline 3 & 2.97 & 8.4 & 28.3 & 273 & 2.99 & 8.6 & 27.9 & 254 \\
\hline 4 & 4.53 & 13.7 & 41.8 & 180 & 3.95 & 11.4 & 35.4 & 229 \\
\hline 6 & 2.76 & 8.9 & 24 & 46 & 2.85 & 8.7 & 24 & 37 \\
\hline 7 & 3.15 & 9.8 & 31.3 & 273 & $/ /$ & // & $/ 1$ & $/ /$ \\
\hline 8 & 2.87 & 9.1 & 25.6 & 25 & 2.79 & 7.7 & 22.1 & 37 \\
\hline 9 & 4.63 & 12.7 & 38.7 & 476 & 4.63 & 12.7 & 38.7 & 476 \\
\hline$\overline{10}$ & 4.14 & 13.2 & 41 & 238 & 3.79 & 12.1 & 36.7 & 237 \\
\hline$\overline{\text { MEDIAN }}$ & 3.59 (IQR2.92-4.43) & 9.8 (IQR 8.9-13.2) & 35 (IQR 26.3-41.6) & 210 (IQR 79.5-264.2) & 3.79 (IQR 2.96-3.975) & 10.15 (IQR 8.675-12.25) & 35.4 (IQR 25.2-38.7) & 200 (IQR 56-237) \\
\hline
\end{tabular}

Table 3.

Descriptive features of included patients treated with endovesical instillation of Vivostat .

\begin{tabular}{|c|c|c|c|c|c|c|c|c|c|c|c|c|c|}
\hline & Age & $\begin{array}{l}\text { Grade of } \\
\text { HC pre }\end{array}$ & $\begin{array}{l}\text { RTOG/ } \\
\text { EORTC pre }\end{array}$ & Primary & $\begin{array}{l}\text { Cause } \\
\text { of HC }\end{array}$ & $\begin{array}{l}\text { Time from } \\
\text { HC onset }\end{array}$ & $\begin{array}{c}\text { Prior intravesical } \\
\text { management }\end{array}$ & $\begin{array}{c}\text { Grade of } \\
\text { hematuria (post-op) }\end{array}$ & $\begin{array}{c}\text { RTOG/ } \\
\text { EORTC post }\end{array}$ & Response & $\begin{array}{c}\text { Hospital } \\
\text { al stay days }\end{array}$ & $\begin{array}{l}\text { ICSI } \\
\text { PRE }\end{array}$ & $\begin{array}{l}\text { ICSI } \\
\text { POST }\end{array}$ \\
\hline 1 & 58 & 2 & 3 & PC & IMRT & & Hyperhydration & 0 & 0 & Complete & 2 & 9 & 5 \\
\hline 2 & 86 & 4 & 4 & PC & IMRT & 1 year & $\begin{array}{l}\text { Bladder irrigation, } \\
\text { Catheterism, } \\
\text { Blood transfusions }\end{array}$ & 0 & 0 & Complete & 3 & 11 & 2 \\
\hline 3 & 75 & 3 & 4 & PC & IMRT & & $\begin{array}{l}\text { Bladder irrigation, } \\
\text { Catheterism, } \\
\text { Blood transfusions }\end{array}$ & $\begin{array}{c}3 \\
1 \text { after } \\
\text { II treatment }\end{array}$ & $\begin{array}{c}3 \\
1 \text { after } \\
\text { II treatment }\end{array}$ & $\begin{array}{c}\text { Clinical } \\
2 \\
\text { instillations }\end{array}$ & 26 & -- & - \\
\hline 4 & 85 & 2 & 3 & PC & IMRT & 11 years & Catheterism & 0 & 0 & Complete & 3 & UC & $U C$ \\
\hline 5 & 27 & 3 & -- & LLA & HSCT & 35 days & Catheterism, Bladder irrigation & 1 & -- & Clinical & 1 & 19 & 1 \\
\hline 6 & 45 & 2 & -- & LH & HSCT & 3 months & Catheterism & 0 & -- & Complete & 1 & -- & -- \\
\hline 7 & 84 & 3 & 4 & PC & IMRT & 5 years & Catheterism, Blood transfusions & -- & -- & --- & 12 & - & -- \\
\hline 8 & 46 & 3 & -- & LMA & HSCT & 20 days & \begin{tabular}{c|} 
Bladder irrigation, \\
Hyperhydration
\end{tabular} & 0 & -. & Clinical & 1 & 15 & 13 \\
\hline 9 & 65 & 3 & 3 & PC & IMRT & 5 years & Catheterism, Bladder irrigation & 0 & 0 & Complete & 4 & $U C$ & $U C$ \\
\hline
\end{tabular}

\section{Results}

A total of 10 patients were treated. The 30\% (n = 3) had HC secondary to bone marrow graft for hematological diseases after systemic chemotherapy treatment, the remaining $70 \%(n=7)$ were diagnosed with actinic cystitis due to prostate cancer RT. The median age was 70 years (IQR 49.0-81.8). In the hematological patients median age was 45 years (IQR 36.0-45.5), while in the radio therapy patients group the median age was 75 years (IQR 70.0-84.5). The median onset time of hematuria was 4.8 (IQR 3.9-6.3) years after RT and 35 (IQR 27.5-62.5) days after hematological treatment. Main preoperative and post-operative laboratory findings are reported in Table 2 .

Of all, 5 patients (55\%) had a complete response (no hematuria or other symptoms), 3 patients (33\%) had only clinical response (improvement or absence of symptoms, grade $<2$ hematuria) and one of them required catheterization and bladder irrigation. Three patients required blood transfusions. One patient died immediately after the loco-regional anesthesia for complications not related to the procedure. Only one patient needed a second FG instillation eleven days after the first one, due to grade 3 hematuria and achieving a clinical response after the second instillation dose. Median hospital stay was 3 (IQR 1-6) days. No adverse events related to the procedure were recorded. Moreover, we recorded a reduction of the median ICSI after treatment. Indeed, the median ICSI was 13.0 (IQR 11.0-15.0) pre-operatively and 4.0 (IQR 2.0$5.0)$ postoperatively $(p=0.02)$. During the median follow up of 12 months, two patients died due to the progression of their hematological condition. None of the others required additional treatment for hematuria. Descriptive features and results of included patients are reported in Table 3.

\section{Discussion}

Hemorrhagic cystitis is a urological condition that can meaningfully affect patients' quality of life. Several ther- 
apeutic options are available for HC treatment, however neither AUA nor EAU defined specific guidelines for the treatment of $\mathrm{HC}(11,17)$. The treatment paradigm includes as first line treatment a noninvasive procedure attempting to reduce hematuria while supporting patients' general condition, such as hyperhydration, blood transfusion, transurethral three-way catheterization, and continuous bladder irrigation (6).

In our study we investigated the results that could be obtained with the use of FG in HC patients' treatment after that first line treatments have failed. Our results showed that a good bleeding control could be achieved with FG endo-vesical instillation with no adverse events, no major complication as ureteral orifice closure and hydronephrosis were recorded in our experience. In case of injection near the ureteral orifice the use of a ureteral open-end catheter is recommended to reduce the risk of iatrogenic ureteral occlusion. Moreover, our results suggest that the use of FG endo-vesical instillation is related with a meaningful improvement in quality of life as proven by the improvement in ICSI score. Moreover, our study showed that the procedure is repeatable and that results are reliable over time as showed by our relative long follow-up of approximately 12 months.

The use of FG as a hemostatic agent have been previously studied in urological surgery and the use of the Vivostat $^{\circledR}$ system have been proven to be effective and safety when applied to urological surgery, in particular to prevent bleeding in renal surgery and lymphatic leakage in lymphadenectomy (18-21). However, only few studies investigated its use for the treatment of HC. Specifically, Bove et al. have investigated the effectiveness of FG treatment in patients with actinic cystitis with hematuria refractory to standard treatment. Their experience was based on a series of 20 patients who developed HC following RT for different types of pelvic cancer: bladder cancer $(30 \%)$, prostate cancer $(35 \%)$ or gynecological cancer (35\%) (13). They concluded that the endoscopic application of FG is a safe and effective therapeutic alternative for the treatment of HC refractory to conventional therapy because FG adheres tenaciously to the damaged mucosa, prevents the worsening of the inflammatory process from urine exposure and allows the tissue repair process. The endo-vesical application during cystoscopy allows a better view of the bleeding points and a better adhesion of the glue to the damaged mucosa due to the constant intravesical pressure (13).

Unfortunately, Bove and colleagues included only patients with actinic cystitis, conversely in the current report we also included patients with HC related to hematological conditions. This is of importance since the $\mathrm{HC}$ related to allogeneic hematopoietic cell transplantation negatively affects patient's quality of life with an increased risk of death $(22,23)$. Currently its therapy was studied by Tirindelli et al. (12) on 35 patients who developed grade $>2$ HC not responsive to conventional therapy. Complete remission, defined as a regression of all symptoms and the absence of hematuria, was achieved in $100 \%$ of the cases at 7 days and $83 \pm 7 \%$ at 50 days from the FG's instillation. The 6-month survival rates of patients with HC was $49 \pm 8 \%$ overall. Instead, if we consider only patients with complete clinical remission the 6-month survival rates were $59 \pm 9 \%$.

The results of this study suggest that the endoscopic application of FG should be considered a safe, non-invasive, easily repeatable, and inexpensive option for the management of $\mathrm{HC}$ in fragile and immunocompromised patients.

When considering other options, hyperbaric oxygen $\mathrm{HBO}$ is a widely used treatment. However, success rates are lower than ours $(34-87.5 \%$ vs. $88 \%$ of our experience) with FG (6). Browne et al. in a literature review reported a successful rate without recurrences of $74 \%$ after $\mathrm{HBO}$. They also reported that the main complication of this treatment was otalgia, occurred in 33\% of patients (7). Intravesical instillation of various substances such as formalin, aluminum salts or hyaluronic acid is also reported. For instance, Pascoe et al. reported a success rate instillation therapy with formalin of 60$90 \%$ vs. $88 \%$ of our experience. However, authors reported serious side effects related to the use of formalin, including death in 2-4\% of the cases. More specifically, Browne et al. also demonstrated the danger of formalin by recording adverse events, such as bilateral hydronephrosis with anuria, vesico-vaginal fistula, and death in $30 \%$ of patients. The same study showed a success rate with hyaluronic acid instillation of 97\% (7). Conversely, we reported no adverse events directly related to treatment. The only death reported was due to the general compromitted status of the patient and not to the FG application.

Aluminum salts were also used. However, its use is considered less effective than other endo-vesical treatment, but it is also affected by side effects: $38 \%$ of patients showed bladder spasms, transient delirium, urinary tract infections. The success rate was $60 \%$ (only $54 \%$ of responders had a durable response).

Imperatore et al. described the use of hyaluronic acid and chondroitin sulfate endo-vesical instillation treatment in 20 patients with refractory Bacillus Calmette-Guerin (BCG) induced chemical cystitis. The study showed an improvement in terms of bladder pain, urinary urgency, urinary volume per void and urinary frequency.

The clinical efficacy described in 19/20 patients had a statistically significative improvement durable in time with a permanence of benefit up to one year after therapy suspension (VAS score on bladder pain and urgency significantly decrease from the baseline; mean number of voids/24 hour and mean urinary volume per void significantly improved from the baseline $\mathrm{p}<0.05$ with respect to baseline in both cases) (24).

In a recent study Masieri et al. analyzed the use of platelet-rich plasma (PRP) in patients with HC after HSCT, they showed an alternative endo-vesical autologous instillation therapy to treat $\mathrm{HC}$ in hematological patients. They studied 10 patients with HC post HSTC related to BK virus infection, all patients underwent PRP instillation after electrocoagulation of the bleeding areas. No intraoperative complications were recorded, postoperative complications Clavien-Dindo Grade II occurred in 6 patients: 3 patients required additional blood transfusion, 3 patients required antibiotic therapy. One patient was readmitted for massive hematuria. 6 patients 
had complete response, 3 partial response and one no response. It seems to be a promising treatment option but related to a higher risk of complication compared to our technique with a similar success rate but in a highly selected group of patients (25).

In summary our study showed that the use of FG in HC patients is safe and effective with higher success rates and lower complications than other second line treatments. Despite the limited number of patients and the lack of a control group, in our study we point out that FG treatment is a promising treatment option also in the group of hematological patients with HC.

The main limitation of our study is the small number of patients included and the absence of a control group. In consequence, we are not able to infer if the use of FG treatment could lead to better result than any other conservative treatment and future prospective multicenter study are warrant. However, the same limitation applies also to other studies that included only few more patients. In addition, due to the small number of patients included we were not able to test the effect of FG treatment on HC in analyses adjusted for possible confounders, such as age or primary cause of HC. It might be possible that the effect of FG might differ based on the baseline characteristics of included patients as a consequence of the compound interaction with the host environment. It is also possible that the coagulation cascade promotion based on FG effect might differ in younger vs. older patients. Future studies should investigate also molecular aspects of FG instillation. Furthermore, we evaluated patients' quality of life with ICSI score. However, other more specific scores about the quality of life in patients affected by malignancy (such as the EPIC score) would be more insightful (26). In future studies it should be included and evaluated to better clarify patients' quality of life. Finally, we investigated only the clinical presentation of $\mathrm{HC}$ without any molecular or laboratory tests that could shed a light on the pharmacodynamic of FG application in HC cases. Indeed, literature is poor of evidence in this field and future studies should focus also on these aspects.

\section{Conclusions}

Autologous FG may be a safe and effective noninvasive and repeatable treatment modality in patients with refractory HC. Our report showed its efficacy also in patients treated after $\mathrm{HC}$ related to hematological conditions treatments and was no related to any adverse event.

\section{AUthors' CONTRIBUtion}

We would like to thank all the Urologists M. Marchioni, F. Silletta, C. D’Orta, G. Primiceri, A. Rizzoli, F Berardinelli, L Schips that recruited the urological patients, performed the surgical procedures and followed them during the follow-up. They also helped with the drafting of this manuscript.

Thanks to the hematologist S. Santarone and A. Natale that recruited and followed the hematological patients during the follow-up, also for the contribution with the processing of the manuscript regarding the hematological therapies and pathologies.
Thanks to the laboratory doctors P. Di Gregorio and S. Verna that helped us with the preparation of the FG solution from patients own plasma. All authors read and approved the final version of the manuscript.

\section{REFERENCES}

1. Hemorrhagic Cystitis - American Urological Association. Available from: https://www.auanet.org/education/auauniversity/ education-products-and-resources/pathology-for-urologists/urinary-bladder/cystitis/hemorrhagic-cystitis.

2. Galla A, Maggio A, Delmastro E, et al. Salvage radiation therapy after radical prostatectomy: survival analysis. Minerva Urol Nefrol. 2019; 71:240.

3. Antonelli A, Palumbo C, Noale M, et al. and the Pros-IT CNR study group. Overview of potential determinants of radical prostatectomy versus radiation therapy in management of clinically localized prostate cancer: results from an Italian, prospective, observational study (the Pros-IT CNR study). Minerva Urol Nefrol. 2020; 72:595-604

4. Corman JM, McClure D, Pritchett R, et al. Treatment of radiation induced hemorrhagic cystitis with hyperbaric oxygen. J. Urol. 2003; 169:2200-2202.

5. Alesawi AM, El-Hakim A, Zorn KC, et al. Radiation-induced hemorrhagic cystitis: Curr. Opin. Support. Palliat. Care. 2014; $8: 235-240$

6. Pascoe C, Duncan C, Lamb BW, et al. Current management of radiation cystitis: a review and practical guide to clinical management. BJU Int. 2019; 123:585-594.

7. Browne C, Davis NF, Mac Craith E, et al. A Narrative review on the pathophysiology and management for radiation cystitis. Adv Urol. 2015; 2015:1-7.

8. Dautruche A, Delouya G. A contemporary review about the management of radiation-induced hemorrhagic cystitis: Curr Opin Support Palliat Care 2018; 12:344-350.

9. Mangano MS, De Gobbi A, Ciaccia M, et al. Actinic cystitis: causes, treatment and experience of a single centre in the last five years. Urol J. 2018; 85:25-28.

10) Calderone CE, Lerner SP, Taylor JM. The case for salvage cystectomy after pelvic radiation. Minerva Urol Nefrol. 2016; 68:161-71.

11. Update Series (2015) Lesson 3: Management of emergency bleeding, recalcitrant clots and hemorrhagic cystitis | AUA University. Available from: https://auau.auanet.org/node/4709.

12. Tirindelli MC, Flammia GP, Bove P, et al. Fibrin glue therapy for severe hemorrhagic cystitis after allogeneic hematopoietic stem cell transplantation. Biol. Blood Marrow Transplant. 2014; 20:1612-1617.

13. Bove P, Iacovelli V, Tirindelli MC, et al. Endoscopic intravesical fibrin glue application in the treatment of refractory hemorrhagic radiation cystitis: a single cohort pilot study. J Endourol. 2019; 33:93-98.

14. Cox JD, Stetz J, Pajak TF. Toxicity criteria of the Radiation Therapy Oncology Group (RTOG) and the European Organization for Research and Treatment of Cancer (EORTC). Int J Radiat Oncol Biol Phys. 1995; 31:1341-1346.

15. Dodd RA, Cornwell R, Holm NE, et al. The Vivostat application system: a comparison with conventional fibrin sealant application systems. Technol Health Care. 2002; 10:401-11.

16. O'Leary MP, Sant GR, Fowler FJ, et al. The interstitial cystitis symptom index and problem index. Urology. 1997; 49:58-63. 
17. Engeler D, Baranowski AP, Berghmans B, et al. EAU Guidelines on Chronic Pelvic Pain 2020. In: European Association of Urology Guidelines. 2020 Edition. European Association of Urology Guidelines Office, 2020, Arnhem, The Netherlands.

18. Schips L, Dalpiaz O, Cestari A, et al. Autologous fibrin glue using the Vivostat system for hemostasis in laparoscopic partial nephrectomy. Eur Urol. 2006; 50:801-805.

(19. Gidaro S, Cindolo L, Lipsky K, et al. Efficacy and safety of the haemostasis achieved by Vivostat system during laparoscopic partial nephrectomy. Arch Ital. Urol Androl. 2009; 81:223-227.

20. Farouk A, Tawfick A, Reda M, et al. Fibrin glue as a sealant in stentless laparoscopic pyeloplasty: A randomised controlled trial. Arab J Urol. 2019; 17:228-233.

21. Garayev A, Aytaç Ö, Tavukcu HH, et al. Effect of Autologous Fibrin Glue on Lymphatic Drainage and Lymphocele Formation in Extended Bilateral Pelvic Lymphadenectomy in Robot-Assisted Radical Prostatectomy. J. Endourol. 2019; 33:761-766.

22. Seber A, Shu X, Defor T, et al. Risk factors for severe hemor- rhagic cystitis following BMT. Bone Marrow Transplant. 1999; 23:35-40.

23. Leung A, Mak R, Lie A, et al. Clinicopathological features and risk factors of clinically overt haemorrhagic cystitis complicating bone marrow transplantation. Bone Marrow Transplant. 2002; 29:509-513.

24. Imperatore V, Creta M, Di Meo S, et al. Intravesical administration of combined hyaluronic acid and chondroitin sulfate can improve symptoms in patients with refractory bacillus CalmetteGuerin induced chemical cystitis: Preliminary experience with oneyear follow-up. Arch Ital Urol Androl. 2018; 90:11-14.

25. Masieri L, Sessa F, Mari A, et al. Intravesical application of platelet-rich plasma in patients with persistent haemorrhagic cystitis after hematopoietic stem cell transplantation: a single-centre preliminary experience. Int Urol Nephrol. 2019; 51:1715-1720.

26. Sosnowski R, Kulpa M, Kosowicz M, et al. Basic methods for the assessment of health-related quality of life in uro-oncological patients. Minerva Urol Nefrol. 2017; 69:409-420.

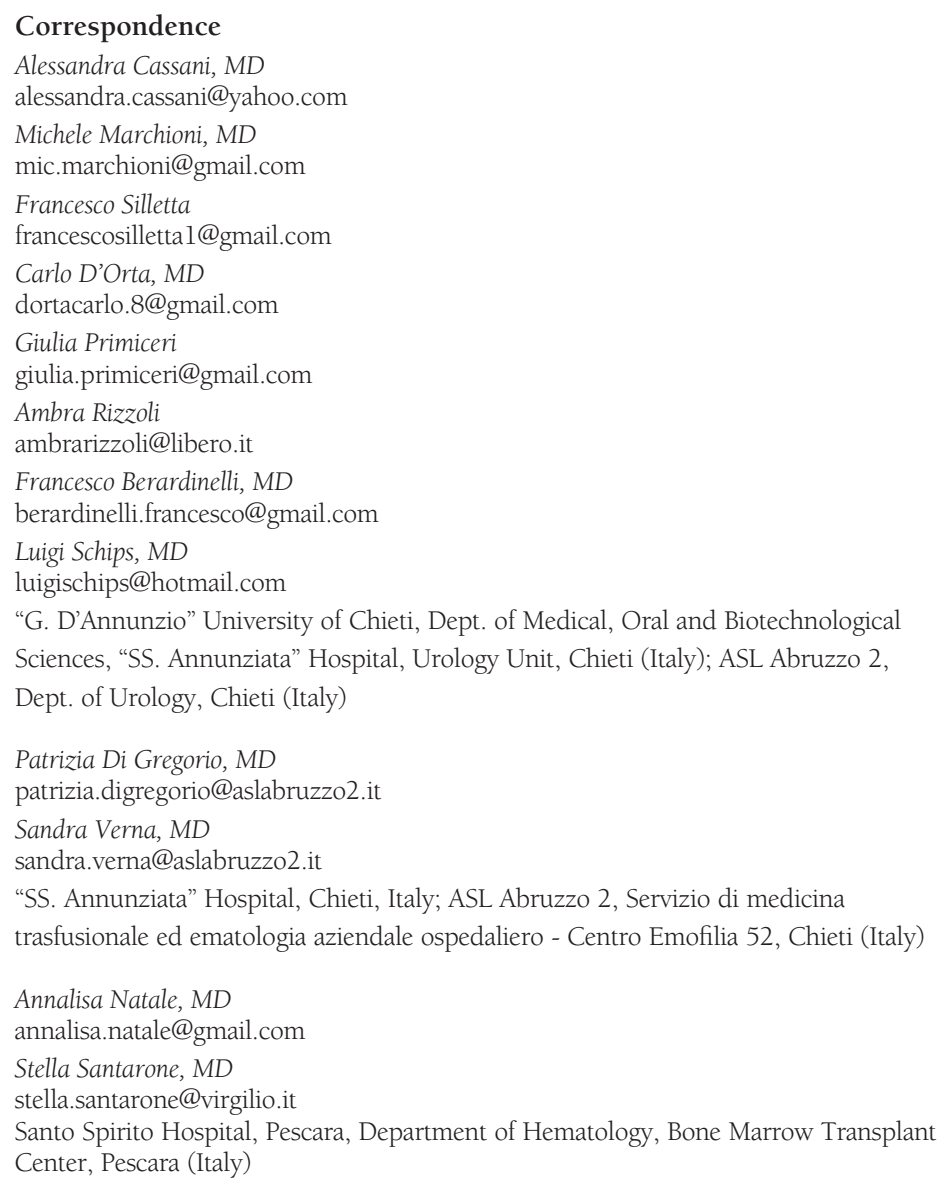

